\title{
An Intelligent Active Queue Management Technique for congestion control
}

\author{
G. Maria Priscilla \\ Asso. Prof. in Computer Science, \\ S.N.R.Sons College (Autonomous), \\ Coimbatore.
}

\begin{abstract}
Congestion an major problem in today's internet traffic had solution with TCP/IP congestion control mechanism. The active queue management (AQM) schemes stabilized the queue oscillations. Earlier RED AQM technique maintained the queue stability in which parameter setting was difficult. Hence a intelligent technique to stabilize the queue in the rapid growing traffic in internet was required. This paper proposes new unsupervised artificial neural network architecture with competitive learning mechanism. Learning vector quantization (LVQ) stabilizes the queue and reduces the queue oscillation. The results are compared with the Kohonen RED (KRED) and Modified Kohonen RED (MKRED) and prove that the proposed LVQ architecture stabilizes queue and maintain the queue delay. Keywords: Active Queue Management, Random Early Detection, neural networks, Kohonen Self Organizing Map, Learning Vector Quantization
\end{abstract}

\section{INTRODUCTION}

In the fastest growing world internet plays a major role in every fields of work. When the flow of traffic is more than the expected level congestion arises as the buffer in the router fails to manage more than the accepted queue length. Packet dropping becomes the solution and results in loss of data. Traditional tail drop mechanism dropped all the packets that arrived above the queue size hence full queue and lock out problem arises. The IETF (Internet engineering task force) recommended active queue management schemes to solve the drop tail issues. Random Early Detection [1] overcame full queue problem and lock out case and had a difficulty in tuning the parameters. The stability of the queue has to be maintained both in heavy traffic flow and in low traffic flow. The adaptive mechanisms of AQM like Feng. RED, adaptive RED and KRED recomputed the $\max _{\mathrm{p}}$. The estimation of setting the parameters in Additive Increase Multiplicative Decrease (AIMD) [2] process are difficult using FRED [3] and ARED [4]. The proposed unsupervised learning neural network architecture computes $\max _{\mathrm{p}}$ value without difficulty achieves an optimal estimation of the probability parameter. This learning method reduces the learning time and stabilizes the queue in both high and low traffic such that congestion is avoided.

\author{
C.P. Sumathi \\ Head \& Asso. Prof. in Computer Science \\ SDNB Vaishnav college for women, \\ Chennai.
}

This paper is structured as follows section II deals with the proposed LVQ architecture. Section III gives the learning methods to train using LVQ and tells about the implementation. Section IV proofs the results with comparison with the earlier architectures KRED [5] and MKRED [6] section V concludes to reach objectives.

\section{ARCHITECTURE}

The architecture of the LVQ [7] net is shown in Fig. 1. There are two layers one input layer and another kohonen layer which are connected with each other. The kohonen layer contains group of neurons and is associated with respective classes. The neurons in the class are equal to $\mathrm{N}$. There are connections between the input layer and the kohonen layer $\mathrm{k}_{\mathrm{i}(\mathrm{i}}$ varies from 1 to $n$ ) carrying node vector called weight vector $\mathrm{w}_{\mathrm{i}}$ [8]; once the training is over every weight vector $\mathrm{w}_{\mathrm{i}}$ belongs to one of the $\mathrm{N}$ prototypes. These prototypes are related to the class which belongs to the neuron $\mathrm{K}_{\mathrm{i}}$. The weights of the neuron get updated as per competitive learning paradigm in which the initial weight is randomly generated. When the input vector $I$ is given to the net to get trained the distance $d_{i}$ is computed between the weight vector $w_{i}$ and I .The Euclidean norm is mostly used to compute the distance between the weight vector and the input vector I

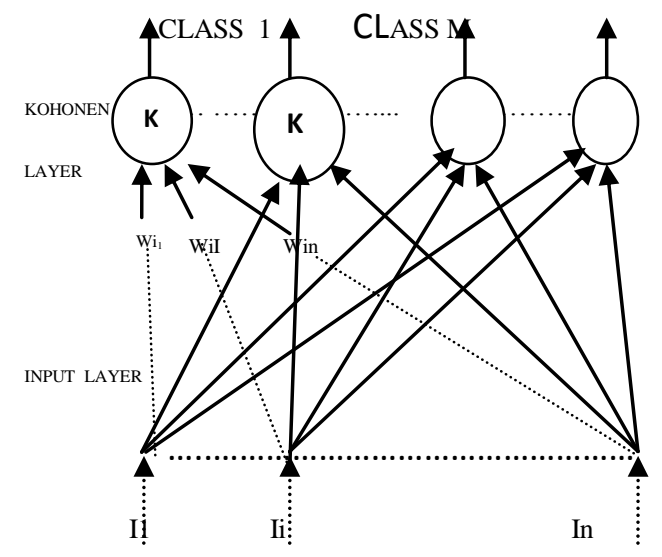

Fig. 1 Architecture of LVQ 


\section{LEARNING METHOD}

The LVQ learns to train the given input vector with weights. First clustering is done by clustering methods to represent data set without class information such that new clusters can also be added and then the classes are labeled by voting method. In the second step the cluster centers are fine tuned to achieve the desired result. A random input vector $\mathrm{x}$ and $\mathrm{a}$ weight vector $\mathrm{w}$ that are close to each other is found. If the selected input vector and weight vector are close of the same class then weight vector is moved towards $\mathrm{x}$ else the $\mathrm{w}$ is moved away from $x$. The LVQ trained with eight long lived TCP/NEWRENO flows generated for 600 seconds. The proposed architecture uses minimum threshold, maximum threshold, queue weight, queue size and maximum probability. he time taken for training is $227 \mathrm{~ms}$ and so no further training has been done. The LVQ algorithm is implemented in ns-2[9] simulator. A matrix of size $25 \times 25$ is used to represent the network.

\section{RESULTS}

This section explains results obtained by LVQ. The dumbbell topology shown in fig 2 . is used for the experiments.

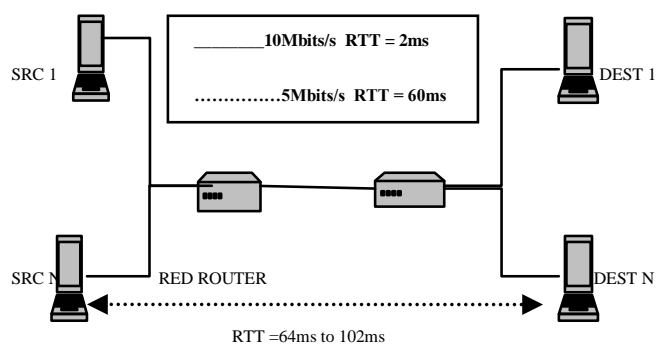

Fig. 2 Dumbbell topology

The obtained results were tested with AIMD streaming data. The traffic here is changed every $50 \mathrm{~ms}$ in order to maintain stability in the queue size The Round Trip Time (RTT) ranges from $64 \mathrm{~ms}$ to $102 \mathrm{~ms}$ which is random for each flow of traffic. The fig 4 explains about the stability of the queue maintained in the various architectures like KRED, MKRED and LVQ ranging between the minimum threshold and the maximum threshold. The training steps compared to KRED, MKRED and LVQ is shown in chart 1. The quality of service parameters like average queue delay, variance of queue delay and packet delivery ratio are discussed in chart 2, chart 3 and chart 4 respectively.

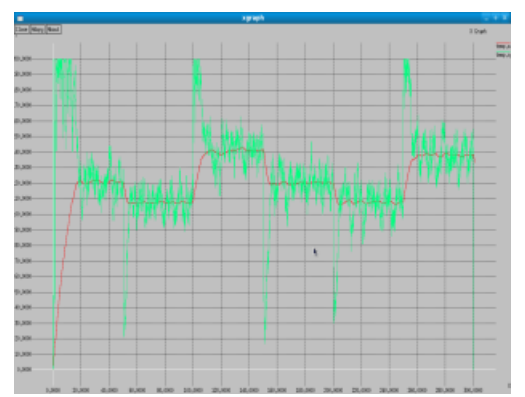

KRED

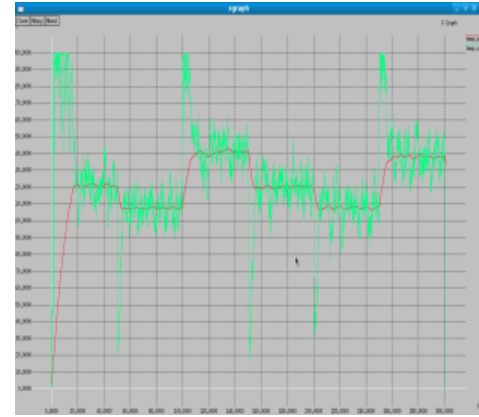

MKRED

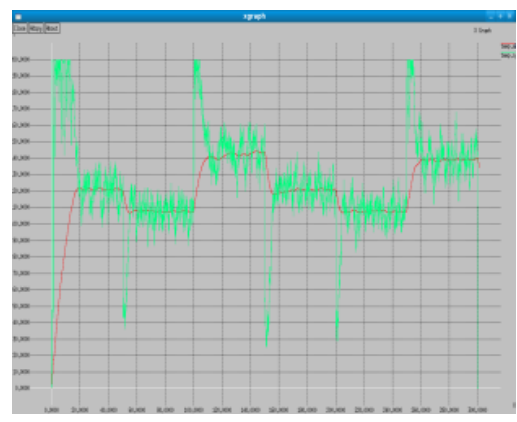

LVQ

Fig 4. Queue Stability with drop rate performance of KRED MKRED \& LVQ

The results are obtained after the training process. The train time received from various architecture is $300 \mathrm{~ms}$ is as shown in the table 1

Table 1. Train step in ms

\begin{tabular}{|c|c|}
\hline Architecture & Time (ms) \\
\hline KRED & 301.4626 \\
\hline MKRED & 248.0127 \\
\hline LVQ & 227.1097 \\
\hline
\end{tabular}

Table 2. Average queue delay in $\mathrm{ms}$

\begin{tabular}{|c|c|}
\hline Architecture & $\begin{array}{c}\text { Queue Delay } \\
(\mathrm{ms})\end{array}$ \\
\hline KRED & 0.003134 \\
\hline MKRED & 0.002252 \\
\hline LVQ & 0.002295 \\
\hline
\end{tabular}


The train time obtained by various architecture to maintain the queue stability is shown in the bar chart 1 .

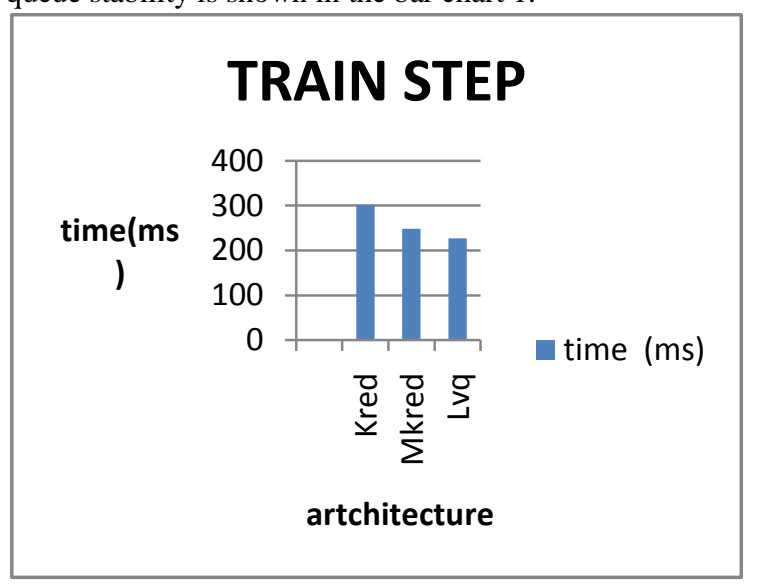

Chart 1: Train step between KRED, MKRED \& LVQ

The average queue length in a bursty traffic is calculated using a low pass filter in an Exponential Weighted Moving Average (EWMA) is

$$
A V G=\left(1-W_{Q}\right) A V G+W_{Q} Q
$$

Where $\mathrm{W}_{\mathrm{Q}}$ determines the time constant if $\mathrm{W}_{\mathrm{Q}}$ is too large the averaging procedure will not filter out transient congestion at the gateway. The average queue size is initially zero and it increases from 0 to $\mathrm{L}$ packets over $\mathrm{L}$ packet arrivals.

The average queue delay for various considered architecture is shown below in table 2 .

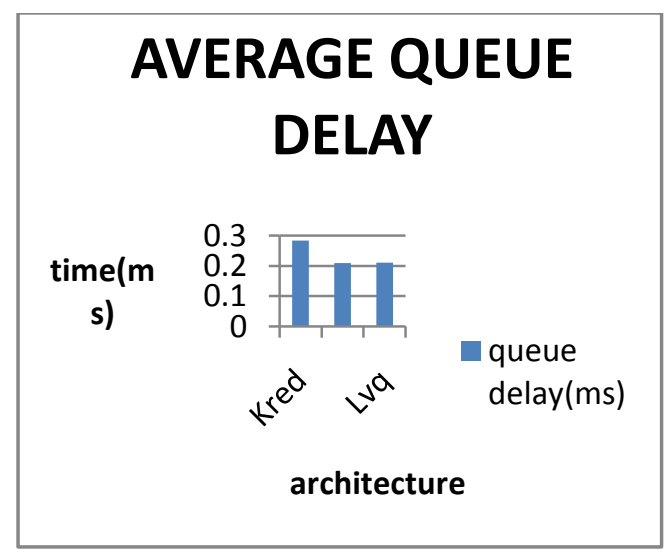

Chart 2: Average Queue Delay between KRED, MKRED \& LVQ

The variance of queue delay called the jitter is shown below in the table 3 .
Table 3. Variance of queue delay

\begin{tabular}{|c|c|}
\hline Architecture & Queue Delay(ms) \\
& 0.283853 \\
\hline KRED & 0.209761 \\
\hline MKRED & 0.210874 \\
\hline LVQ & \\
\hline
\end{tabular}

The packet delivery ratio is calculated as follows $\quad$ PDR $=$ bytes received /packets sent

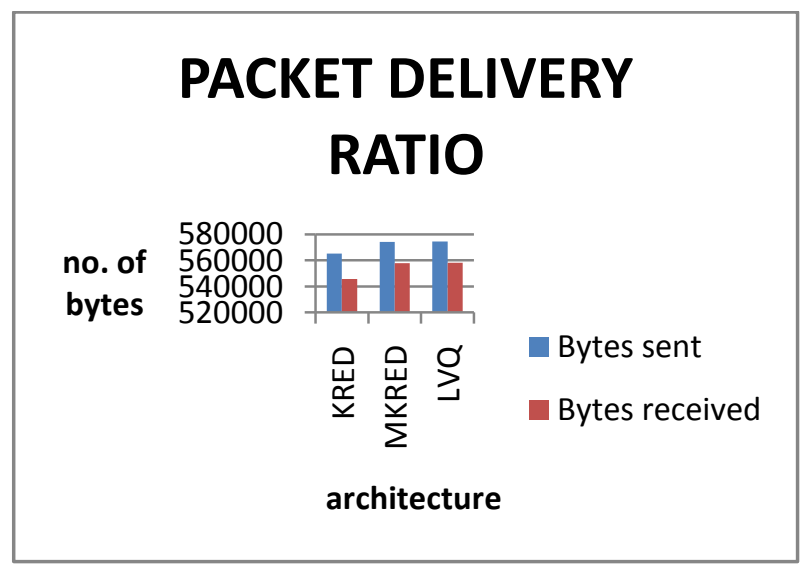

Chart 3 : Variance of Queue Delay between KRED, MKRED \& LVQ

Table 4 . Packet delivery ratio

\begin{tabular}{|c|c|c|c|}
\hline Architecture & $\begin{array}{c}\text { Bytes } \\
\text { sent }\end{array}$ & $\begin{array}{c}\text { Bytes } \\
\text { received }\end{array}$ & PDR \\
\hline KRED & 565365 & 545740 & 0.9731 \\
\hline MKRED & 574247 & 557947 & 0.9716 \\
\hline LVQ & 574606 & 558134 & 0.9713 \\
\hline
\end{tabular}




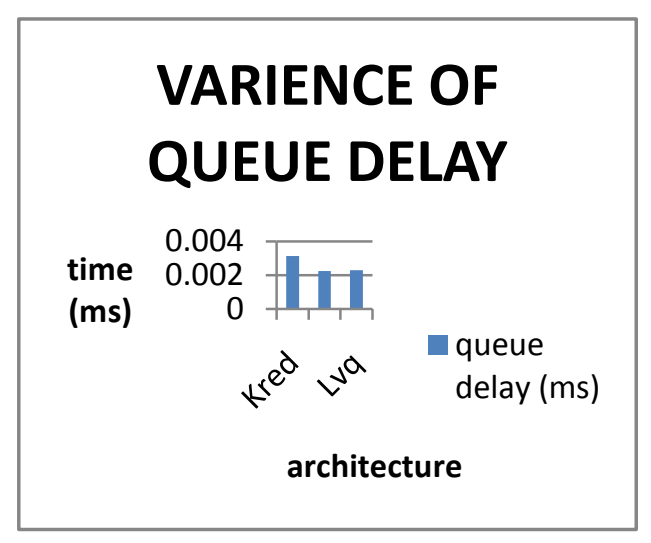

\section{Chart 4: Packet Delivery Ratio between KRED, MKRED \& LVQ}

\section{DISCUSSION AND CONCLUSION}

The proposed architecture which is an unsupervised competitive learning vector quantization mechanism stabilizes the queue and gives better performance in the average queue delay, variance of queue delay. The training steps obtained are very minimum compared to the already used KRED and MKRED architectures. LVQ computes maximum probability without any difficulty in setting parameters and is implemented in ns-2. LVQ has been trained well and does not require retraining so it well suit to be put in the hardware in the router. The experiment results show that the proposed algorithm is efficient in stabilizing the queue for the heavy traffic and low traffic intelligently.
[1] S. Floyd and V. Jacobson, "Random early detection gateways for congestion avoidance," IEEE/ACM Trans. Networking, vol. 1, no. 4, pp. 397-413, Aug. 1993.

[2] Yang Richard Yang and Simon S. Lam "General AIMD Congestion Control "Proceedings of ICNP 2000, Osaka , Japan , November 2000.

[3] Wu-chang Feng, "Improving internet congestion control and queue management algorithms ".

[4]. Rahul Verma, Aravind Iyer and Abhay Karandikar "Active Queue Management using Adaptive RED" Journal of communications and networks july 152002

[5] Emmanuel Lochin and BrunoTalavera "Managing network congestion with a Kohonen-based RED queue" ICC 2008 proceedings

[6] G. Maria Priscilla and Antony Selvadoss Thanamani,"managing network congestion with a modified

Kohonen based RED queue", International Journal of Engineering Science and Technology Vol. 2(11), 2010, 6747-6752

[7] T. Kohonen. Learning vector quantization. In: M.A. Arbib, editor, The Handbook of Brain Theory and Neural Networks., pages 537-540. MIT Press, Cambridge, MA, 1995.

[8] Kohonen, T., The Self-Organizing Map, Proceedings of the IEEE, Vol.78, No.9,1990, pp. 1464-1480.

[9] Arijit Ganguly and Pasi Lassila "A study of TCP-RED congestion control using ns2"

[10] Dina Goren-Bar, Tsvi Kuflik, Dror Lev “supervised learning for automatic classification of documents using self- organizing maps"

[11] Vincent Cheung and Kevin Cannons "An introduction to neural networks "

[12] Chrysostomos Koutsimanis and Pan Gan Park "Active Queue Management - A router based control mechanism

\section{REFERENCES}

